\title{
Diseño y Evaluación de una Planta de Reciclaje de Envases Tetra Pak a Pequeña Escala
}

\author{
Recepción: Noviembre de 2004 / Aceptación: Diciembre 2004
}

\author{
(1) Jorge Inche Mitma \\ (2) Jorge Vergiú Canto \\ (3) Daniel Mavila Hinojoza \\ (4) Manuel Godoy Martínez \\ (5) Alfonso Chung Pinzás
}

\section{RESUMEN}

El estudio trata sobre el diseño y evaluación de una planta de reciclaje de envases tetra pak a pequeña escala. A partir de la información recopilada, se encontró la ingeniería básica de la planta, incluyendo los aspectos de: diseño del producto, diseño del proceso, diseño de equipos y evaluación económica; a fin de establecer la factibilidad técnica, económica y ambiental para su implementación.

Palabras Claves: Reciclaje. Diseño de planta. Envases de cartón.

Designand Evaluation of Tetra-pak Containers' Recycling Plant on a S Mall S Cale ABSTRACT

This study deals about the design and evaluation of Tetra Pak containers' recycling plant on a small scale. The basic Plant Engineering was found from the information gathered. Some aspects included were: product design, process design, equipment design and costs evaluation, with the aim of determining its technical, economical and environmental capability for its implementation.

Key words: Recycling. Plant design. Carton containers.

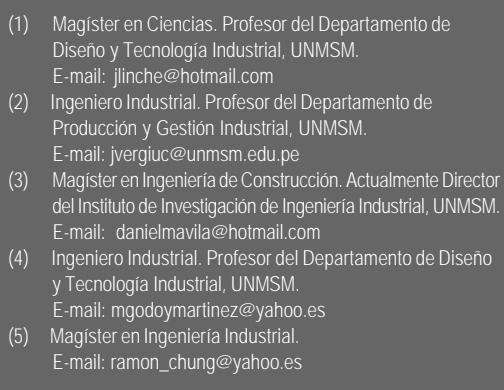

\section{INTRODUCCIÓN}

El estudio se inicia con los resultados de la investigación sobre el desarrollo de un prototipo a partir de envases reciclados (Rev. Industrial Data, Vol. 6, Nro.2, 2003).

Se recopiló información de iniciativas desarrolladas por CEPIS, PNUMA y la Municipalidad de Surco, en relación al reciclaje de residuos provenientes de los envases de Tetra Pak.

En China y Alemania se han establecido experiencias en materiales, Ilamados "tectán", utilizados en la industria del mueble y de la construcción.

\section{MARCO CONCEPTUAL}

\section{Antecedentes}

El uso del TECTAN está difundido en Europa, en especial en Alemania esto gracias al impulso dado por la propia empresa TETRA PAK, asimismo en China en donde se le conoce como CHIPTEC, cuenta con 3 fábricas de CHIPTEC y la Agencia China de Protección Ambiental desde 1997 reconoce al CHIPTEC como "una de las tecnologías recomendables, a escala nacional, para la protección del medio ambiente", otro caso es el de Chile donde hubo un programa denominado "Un Techo para Chile" el cual impulsó la construcción de aldeas con este material, esto gracias al CONAMA de Chile y al Hogar de Cristo.

En el caso peruano recién está ingresando esta tecnología, gracias a la misma empresa Tetra Pak; sin embargo aún es incipiente. Primero, se hizo un trabajo de campo recorriendo los principales centros de venta de residuos segregados preguntando si se conocía el envase de Tetra Pak y si era así, cuánto de este producto se vendía como material de reciclaje, las respuestas fueron negativas, el $100 \%$ de los encuestados respondieron que no conocían el envase de Tetra Pak. Por otro lado, enseñándoles la muestra se continuó preguntando si es que se recicla este producto, a lo cual respondieron que no comercializan dicho producto.

La segunda parte de la investigación de los antecedentes se llevó a cabo en la Planta de Reciclaje de la Municipalidad de Santiago de Surco, en donde se entrevistó al gerente de dicha planta el General (r) Luis Carlos Baluarte Guevara y manifestó que los envases de Tetra Pak no se venden ya que las empresas no los compran y que al mes segregan cerca de 4 TM de Tetra Pak, los cuales son trasladados al relleno sanitario. 


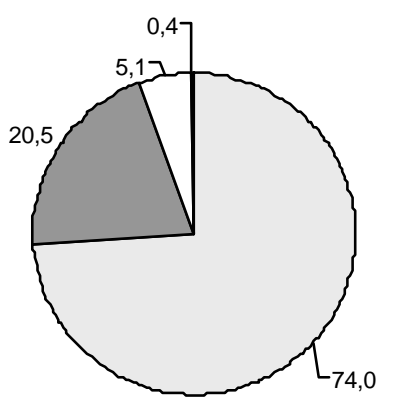

Figura 1. Composición del Tetra Pak Fuente: Empresa Tetra Pak, 2002.

Caracterización de Envases Reciclados El uso de la marca Tetra Pak está íntimamente relacionado con el producto "estrella" de esta empresa, en realidad Tetra Pak es el nombre de la empresa y no de los envases. Sin embargo, todo el mundo identifica estos envases con el nombre de la empresa.

Las plantas para Sudamérica se encuentran ubicadas en Colombia y Perú y sólo venden el producto Tetra Pak. Este envase tiene una lámina gruesa compuesta de aluminio, cartón y polietileno, cuya composición se muestra en la Figura 1.

Según datos de proyecciones de la Supervisión Municipal de Servicios de Limpieza (SUMSEL), en el año 2000, Lima Metropolitana genera 1390242 toneladas, de estas cantidades sólo el $51,58 \%$ se dispone en los rellenos sanitarios, y del resto no se conoce el destino final. Tal como se muestra en el Cuadro 1.

En cuanto a la composición de los residuos sólidos, se tiene información para el año 2000 de la Dirección General de Salud (DIGESA), donde no figura el residuo tetrapak (ver Figura 2).

Con la intención de cuantificar el contenido de envases tetra pak dentro de la basura urbana, se hizo un

Cuadro 1. Generación estimada de residuos en Lima Metropolitana (2000)

\begin{tabular}{|c|c|c}
\hline Distrito & $\begin{array}{c}\text { Generación } \\
\text { percápita } \\
\text { (Kg/habitante/año) }\end{array}$ & $\begin{array}{c}\text { Generación } \\
\text { (TM/año) }\end{array}$ \\
\hline Surco & 0,840 & 75137 \\
$\begin{array}{c}43 \text { distritos de } \\
\text { Lima } \\
\text { Metropolitana }\end{array}$ & 0,567 & 1390242 \\
\hline
\end{tabular}

Fuente: Proyecciones de la Supervisión Municipal de Servicios de Limpieza (SUMSEL). Instituto Cuanto, 2001. muestreo preliminar en 5 puntos del Distrito de Santiago de Surco, obteniéndose $6,88 \%$ de tetra pak. Asimismo, los envases de tetra pak en su composición contienen mayor cantidad de polietileno y papel.

Para entender mejor el motivo de la investigación y los resultados es necesario comprender el origen de los envases Tetra Pak.

\section{a. La empresa Tetra Pak}

La empresa Tetra Pak es una transnacional, se fundó en el año 1952 en Suecia gracias a la idea del Dr. Rubén Rausing sobre un novedoso envase de leche el cual podía conservar su contenido durante meses sin conservante ni refrigeración.

Para entender mejor la filosofía de trabajo de esta compañía se transcribe la visión y la misión ambas extraídas de su página web www.tetrapak.com.pe:

Visión:

«Nos comprometemos a hacer los alimentos seguros y disponibles en todas partes».

\section{Misión:}

«Trabajamos por y con nuestros clientes para proporcionar las soluciones preferidas de tratamiento y envasado de alimentos. Aplicamos nuestro compromiso con la innovación, nuestro entendimiento de las necesidades del consumidor y nuestra relación con los proveedores para brindar soluciones cuando y donde se consuman alimentos . Creemos en un liderazgo industrial responsable, que apuesta a un crecimiento rentable en armonía con sustentabilidad ambiental y civismo empresarial».

Si se analiza detenidamente la Visión y Misión, se llega a la conclusión de que en efecto responden a la fabricación de productos envasados en las mejores condiciones conforme al requerimiento del consumidor.

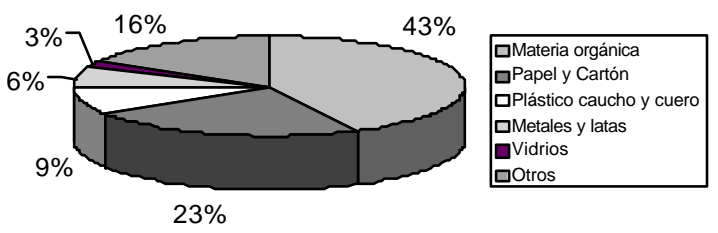

Figura 2. Composición de residuos sólidos en Lima Metropolitana Fuente: Ministerio de Salud. DIGESA. 2000 
El envase "Tetra Classic" fue un invento "revolucionario" en su época ya que utilizaba menos material de envase que cualquier otro diseño de cartón, y el material estaba cubierto de plástico en lugar de cera, que era el material utilizado por la mayoría de los envases de ese entonces. Asimismo, la forma de llenar los envases también resultó novedosa, a diferencia de los envases tradicionales de aquel entonces, estos eran alimentados en rollos de cartón y se utilizaban máquinas de formar/llenar/sellar, cerrando herméticamente cada envase.

En el año 1961, la compañía Tetra Pak lanzó un nuevo producto, denominado envase de cartón aséptico; es decir, se incorporó al envase una lámina gruesa de aluminio como barrera y un tratamiento de esterilización de corta duración pero a alta temperatura. Todo esto colocó a la empresa a la vanguardia en lo que a ciencias alimentarias se refiere, tanto así que lo denominaron "el avance más importante en la ciencia de alimentos desde los tiempos de Pasteur"; por otro lado el Instituto de Tecnólogos de Alimentos (Institute of Food Technologists, IFT), en su publicación del año 1989 lo ubicó dentro de las 10 mejores innovaciones en las ciencias alimentarias debido a la seguridad que proporciona a los alimentos.

\section{Madera Sintética como Alternativa}

La madera sintética obtenida de los residuos de los envases Tetra Pak, se denomina Tectan (ver Figura $3)$, por ser el más conocido de todos los diferentes nombres que recibe en diferentes partes del mundo (Chiptek, Maplar, etc.), su producción está muy difundida en Europa. En cuanto al Perú, aún no se recicla a nivel comercial.

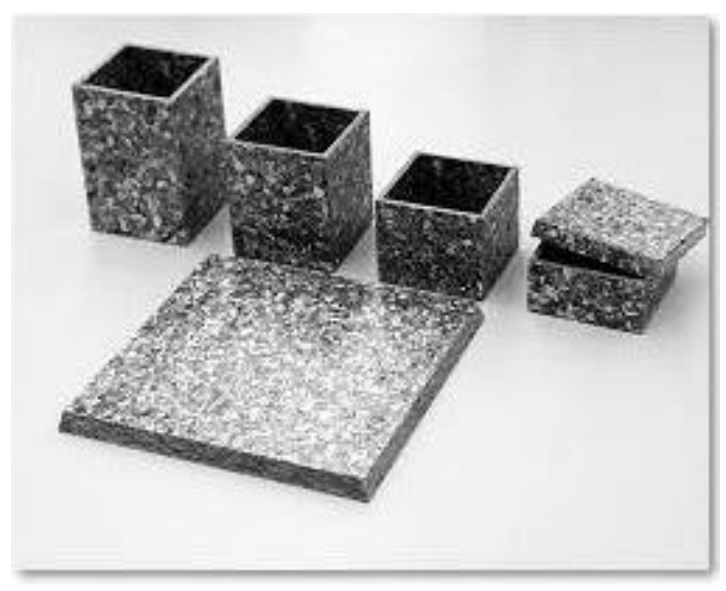

Figura 3. Planchas de Tectán Fuente: Empresa Tetra Pak, 2002.
Los artículos que se pueden fabricar con estas planchas son variados tales como: separadores de ambientes, muebles, carpetas etc.

\section{Diseño de Planta}

El factor principal que da lugar a la decisión de fabricación de un nuevo producto, o a la expansión o modernización de las condiciones presentes, es generalmente de tipo económico y suele ir acompañado de esta pregunta: ¿Cuáles serán las utilidades de esta inversión?. El ingeniero de diseño debe estar en una posición tal que le sea posible dar a la gerencia unos costos de preconstrucción estimados, basados en un diseño preliminar de la planta para la fabricación del producto, para que sea posible llegar a una decisión con bases firmes. Este análisis de diseño de planta preliminar incluye:

- Proceso de fabricación.

- Selección del equipo y materiales del proceso.

- Distribución de planta y consideraciones en cuanto a la localización.

- Costo de fabricación.

\section{MÉTODOS Y RESULTADOS}

\section{Método}

El diseño de planta detallado comprende la siguiente secuencia de pasos:

a. Ubicación de la planta.

b. Obtención de datos metereológicos y de sismicidad.

c. Diseño del producto: Características y atributos del producto.

d. Diseño del proceso: Etapas del proceso, materias primas, productos intermedios, subproductos, residuos y productos finales. Diagrama de flujo del proceso.

e. Diseño del equipo: Determinar el equipo y la maquinaria necesarios para la fabricación, en función del tipo de producto o productos.

f. Fijar el número de unidades de cada máquina y tipo de equipo necesarios para fabricar cada producto en función del volumen de ventas.

g. Calcular el espacio total requerido para la planta, sumando:

- El espacio necesario para la maquinaria.

- Área de desenvolvimiento del operario.

- Área para el servicio a las máquinas.

- Área para acceso y salida de materiales.

- Área para productos terminados.

- Área para servicios al personal.

- Área para parqueo y áreas verdes. 
h. Distribuir los diferentes departamentos en sus respectivas zonas de trabajo, de modo que el recorrido del trabajo sea lo más económico posible.

i. Establecer la distribución de planta, teniendo en cuenta sobre todo la ubicación de las zonas de trabajo, áreas de almacenamiento y servicios auxiliares.

j. Determinar el tamaño y disposición del terreno exterior a la fábrica, asignando el espacio necesario para estacionamiento, recepción, embarque y áreas verdes.

k. Determinar el costo de equipos, terreno y edificación.

I. Determinar el costo de la planta, inversiones e indicadores económicos financieros.

\section{Resultados}

a. Ubicación de la planta

La Planta de Reciclaje se ubicará en la Urbanización Entel Perú, del distrito de San Juan de Miraflores, en la provincia de Lima. El plano de ubicación se muestra en la Figura 4.

Las coordenadas geográficas de ubicación de la planta se estableció con el receptor GPS (Sistema de Posición Global), obteniéndose la siguienteinformación:

Altitud: $129 \mathrm{msnm}$

Latitud: $12 \circ$ (Norte y Sur)

Longitud: $77^{\circ}$ (Este y Oeste)

b. Datos metereológicos Invierno: Temperatura debajo de $20^{\circ} \mathrm{C}$ con neblina por 6 ú 8 meses.

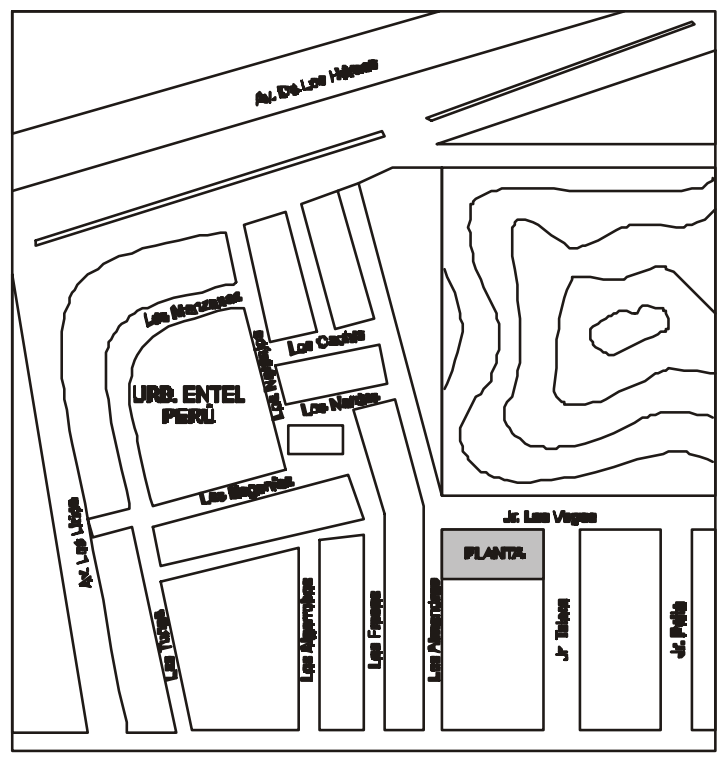

Figura 4. Plano de ubicación de la planta Fuente: Elaboración propia, 2004.
Verano: Temperatura encima de $28{ }^{\circ} \mathrm{C}$ con alta humedad.

Promedio de Precipitación Anual (mm): 20

Velocidad del Viento $(\mathrm{km} / \mathrm{h}): 0,9$

Dirección del Viento: SN

Promedio de Humedad Relativa (\%): 80

Promedio presión barométrica (KPa): 97,3

c. Datos de zona sísmica

A continuación se muestra los eventos sísmicos que han ocurrido desde 1940. El riesgo moderado se presenta a partir de 1966.

\begin{tabular}{|c|cc|c|}
\multirow{2}{*}{ FECHA } & \multicolumn{2}{|c|}{ COORDENADAS } & ESCALA DE \\
& Latitud & Longitud & RITCHER \\
\hline 24.05 .1940 & 11.2 & 77.5 & 8.2 \\
17.10 .1966 & 10.7 & 78.7 & 7.5 \\
31.05 .1970 & 9.2 & 78.8 & 7.5 \\
03.10 .1974 & 12.05 & 77.4 & 7.6
\end{tabular}

d. Diseño del producto

El producto final consiste en planchas de dimensiones $1,2 \times 2,4 \times 0,01 \mathrm{~m}$.

Los atributos del producto, son:

- Permite una construcción sólida y duradera.

- Reciclaje 100\%.

- No incorpora productos tóxicos ni peligrosos.

- Puede ser aserrado, mecanizado, clavado y encolado.

- No se astilla ni se agrieta.

- No conduce la electricidad, además de producir un aislamiento térmico y acústico.

- Insensible a la putrefacción, insectos y hongos.

Las características técnicas del producto se muestran en el Cuadro 2.

e. Diseño del proceso

- Recolección

En el estudio, la materia prima se obtuvo de la planta de segregación de residuos de la Municipalidad Distrital de Surco.

- Separación y limpieza

Se realiza en forma manual, escogiendo los envases de tetra pak vacíos, su objetivo es, eliminar los residuos líquidos existentes en algunos envases usados.

- Molienda

La molienda se lleva a cabo por trabajo mecánico, aplicando fuerzas de tensión, compresión y corte. Esta operación permite la obtención de pequeños fragmentos cercanos a $3 \mathrm{~mm}$. 


\section{Cuadro 2. Características del producto}

\begin{tabular}{|c|c|c|}
\hline Item & Característica & Calificación \\
\hline 01 & Densidad & $800-900 \mathrm{Kg} / \mathrm{m}^{3}$ \\
\hline 02 & Módulo de Rotura* $\left(\mathrm{N} / \mathrm{mm}^{2}\right)$ & 14,95 \\
\hline 03 & Módulo de elasticidad* $\left(\mathrm{N} / \mathrm{mm}^{2}\right)$ & 1050 \\
\hline 04 & Absorción de agua a 24 horas & $<1,0 \%$ \\
\hline 05 & Hinchazón de agua a 24 horas & $<0,8 \%$ \\
\hline 06 & $\begin{array}{l}\text { Comportamiento frente a } \\
\text { ambiente marino }\end{array}$ & Sin deterioro \\
\hline 07 & $\begin{array}{l}\text { Estabilidad dimensional frente a } \\
\text { cambios de humedad (longitud) }\end{array}$ & $<0,5 \%$ \\
\hline 08 & $\begin{array}{l}\text { Resistencia química } \\
\text { (detergente, lejía, HCL) }\end{array}$ & Muy buena \\
\hline 09 & $\begin{array}{l}\text { Estabilidad longitudinal frente a } \\
\text { cambios de temperatura, } 24 \\
\text { horas a } 70 \stackrel{\circ}{ } \mathrm{C}\end{array}$ & $0,05 \%$ \\
\hline$\overline{10}$ & $\begin{array}{l}\text { Comportamiento frente al } \\
\text { ataque biológico }\end{array}$ & Sin deterioro \\
\hline 11 & $\begin{array}{l}\text { Resistencia al arranque de } \\
\text { tornillos }\end{array}$ & $<1,625 \mathrm{~N}$ \\
\hline 12 & Resistencia al impacto & Muy buena \\
\hline 13 & Mecanizado: cortar, clavar, & Muy bueno \\
\hline
\end{tabular}

- Prensado

El material triturado se extiende en una capa de aproximadamente $10 \mathrm{~cm}$. Después se somete a compresión mediante una prensa hidráulica a $170^{\circ} \mathrm{C}$. El calor funde el contenido de polietileno (PE) que une la fibra densamente comprimida y los fragmentos de aluminio en una matriz elástica.

- Enfriado

La matriz resultante se enfría después rápida- mente, formando un duro aglomerado con una superficie brillante e impermeable. El polietileno es un agente de unión muy eficaz, de manera que no es necesario añadir cola u otros productos químicos.

f. Diseño del equipo

- Trituradora de cuchillas

Está diseñada específicamente para soportar una gran carga de materiales para su triturado. El cuerpo de la maquinaría está fabricado en hierro y acero reforzado. Su motor, con cuchillas de acero, se encuentra protegido contra partículas de polvo, puede triturar toda clase de materiales de plástico. Las cuchillas de esta trituradora, funcionan de manera similar a tijeras haciendo simple el triturado, sin la necesidad de pulverizar ni de utilizar calor. Las dos bocas de alimentación de material, se encuentran convenientemente ubicadas para una fácil disposición del material a pulverizar (ver Figura 6 y Cuadro 3).

\section{- Prensa Hidráulica}

Ocupa un amplio campo de la industria de la transformación, esto se debe a las múltiples posibilidades de reglajes que ofrece el mando electrohidráulico, ya que se puede adaptar la máquina al proceso de transformación, a las características del material y a su configuración (ver Figura 7). Por su diseño, el bastidor de la prensa puede absorber cargas muy elevadas con una deformación mínima, y así

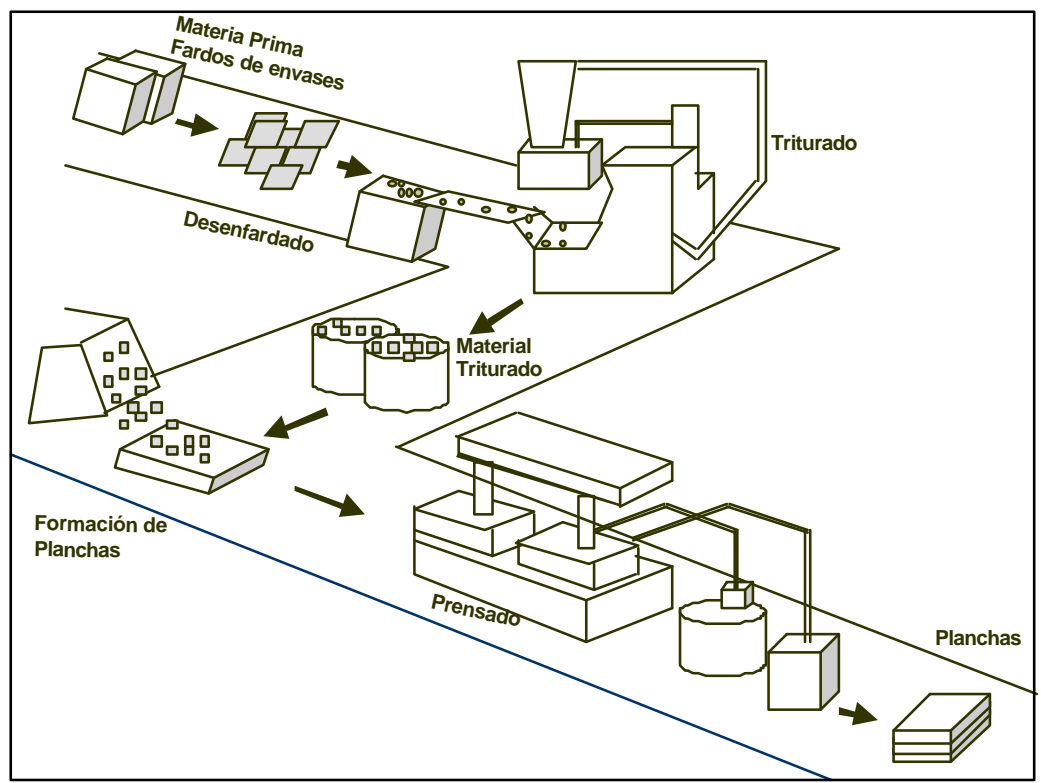

Figura 5. Proceso de fabricación del tectán 
Cuadro 3. Características de la trituradora de cuchillas

\begin{tabular}{|c|l|c|}
\hline Item & Característica & Especificación \\
\hline 01 & Volumen - Tolva $\left(\mathrm{m}^{3}\right)$ & 6 \\
\hline 02 & Superficie de trabajo $(\mathrm{mm})$ & $1500 \times 1100$ \\
\hline 03 & $\mathrm{~N}^{\circ}$ de árboles & 2 \\
\hline 04 & $\mathrm{~N}^{\circ}$ de árboles $(\mathrm{RPM})$ & $13 / 8$ \\
\hline 05 & $\mathrm{~N}^{\circ}$ de cuchillas de $75 \mathrm{~mm}$ & 20 \\
\hline 06 & $\mathrm{~N}^{\circ}$ Cuchillas de $100 \mathrm{~mm}$ & 15 \\
\hline 07 & Potencia de motor $(\mathrm{KW})$ & 75 \\
\hline 08 & Tensión $(\mathrm{V})$ & $220 / 380 / 440$ \\
\hline 09 & Corriente $(\mathrm{A})$ & $240 / 140 / 120$ \\
\hline 10 & Peso $(\mathrm{Kg})$ & 13000 \\
\hline
\end{tabular}

realizar piezas de gran precisión. El prensado se efectúa en calientep ara las planchas de tetra pak. Para ello, los moldes están en contacto con la resistencia eléctrica que genera calor.

La prensa hidráulica tiene la siguiente especificación:

- Tonelaje: 150

- Mesa: 2050 x $800 \mathrm{~mm}$.

- Peso: 10Toneladas.

- Mandos: Manual y con pedal.

g. Superficie de la planta

La superficie de los equipos se determina sumando los siguientes rubros: la superficie del equipo, área de desenvolvimiento del trabajador y superficie de almacenamiento de entrada y salida, dando por resultado $43 \mathrm{~m}^{2}$, conforme al Cuadro 4. La superficie de instalaciones y servicios auxiliares resulta $208 \mathrm{~m}^{2}$, conforme al Cuadro 5.

Por consiguiente la superficie de la planta incluye los componentes de los cuadros 4 y 5 , resultando una superficie total de $251 \mathrm{~m}^{2}$. Asimismo, la distribución de planta (con un $13 \%$ de sobredimensionamiento) se muestra en la Figura 8.

h. Evaluación económica de la planta

- Inversión inicial

Comprende la inversión fija tangible e intangible más el capital de trabajo, mostrado en el Cuadro 6.

- Costo de producción

Incluye los costos de materiales directos e indirectos, mano de obra directa e indirecta y gastos generales de fabricación (depreciación y servicios), mostrados en los cuadros 7, 8, 9, 10 y 11.

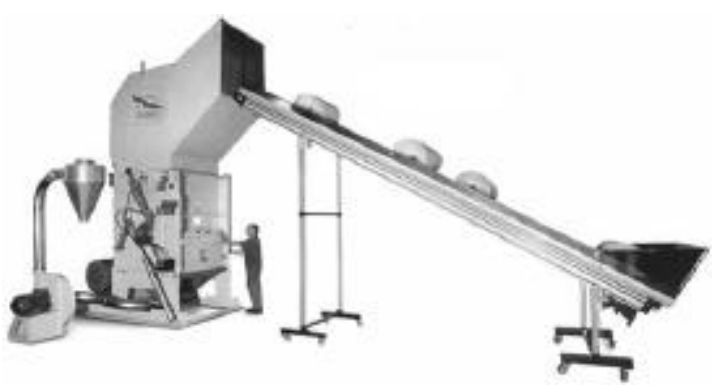

Figura 6. Trituradora de cuchillas

- Costo total anual

Incluye el costo de producción más los costos de administración obtenidos de los cuadros 12,13 y 14 , tal como se muestra en el Cuadro 15.

- Depreciación total Incluye la depreciación de los costos indirectos de producción y la depreciación de los costos administrativos, mostrados en el Cuadro 16.

- Ingresos

De acuerdo a la capacidad de planta se estima una producción de 126 planchas/día generando un ingreso anual de 226800 dólares (ver Cuadro 17).

- Flujo de caja

A partir del flujo de caja (ver Cuadro 18) el estudio se evalúa con el Valor Actual Neto

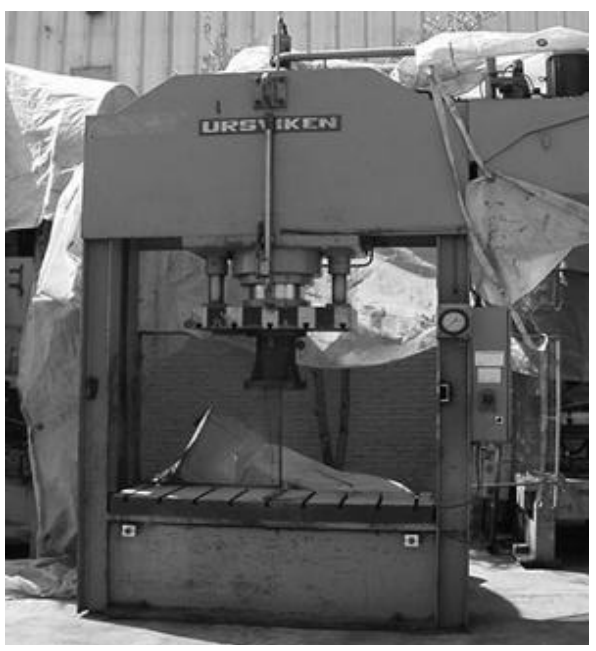

Figura 7. Prensa hidraúlica 
Cuadro 4. Superficie de los equipos

\begin{tabular}{|l|c|c|c|c|}
\hline Máquina/Equipo & $\begin{array}{c}\text { Superficie del } \\
\text { equipo }\left(\mathrm{m}^{2}\right), \\
\text { incluye mesa } \\
\text { de trabaio }\end{array}$ & $\begin{array}{c}\text { Área de } \\
\text { desenvolvi- } \\
\text { miento }\end{array}$ & $\begin{array}{c}\text { Superficie de } \\
\text { almacenamiento } \\
\left(\mathbf{m}^{2}\right) \text {, incluye lugar } \\
\text { para herramientas } \\
\text { v utensilios }\end{array}$ & $\begin{array}{c}\text { Superficie } \\
\text { total }\left(\mathrm{m}^{2}\right)\end{array}$ \\
\hline Prensa hidráulica (2) & 12 & 4 & 2 & 18 \\
\hline $\begin{array}{l}\text { Trituradora de } \\
\text { cuchillas }\end{array}$ & 7 & 4 & 3 & 14 \\
\hline Sierra circular & 2 & 2 & 3 & 7 \\
\hline $\begin{array}{l}\text { Intercambiador de } \\
\text { calor }\end{array}$ & 2 & -- & 2 & 4 \\
\hline
\end{tabular}

Cuadro 5. Superficie de instalaciones y servicios auxiliares

\begin{tabular}{|lc}
\hline \multicolumn{1}{|c}{ INSTALACIONES } & $\begin{array}{c}\text { SUPERFICIE } \\
\left(\mathbf{m}^{2}\right)\end{array}$ \\
\hline Almacenamiento de materia prima & 40 \\
\hline Almacenamiento de producto terminado & 40 \\
\hline Taller de mecánica & 20 \\
Oficinas & 48 \\
Servicios higiénicos & 20 \\
\hline Playa de estacionamiento & 40 \\
\hline TOTAL & 208 \\
\hline
\end{tabular}

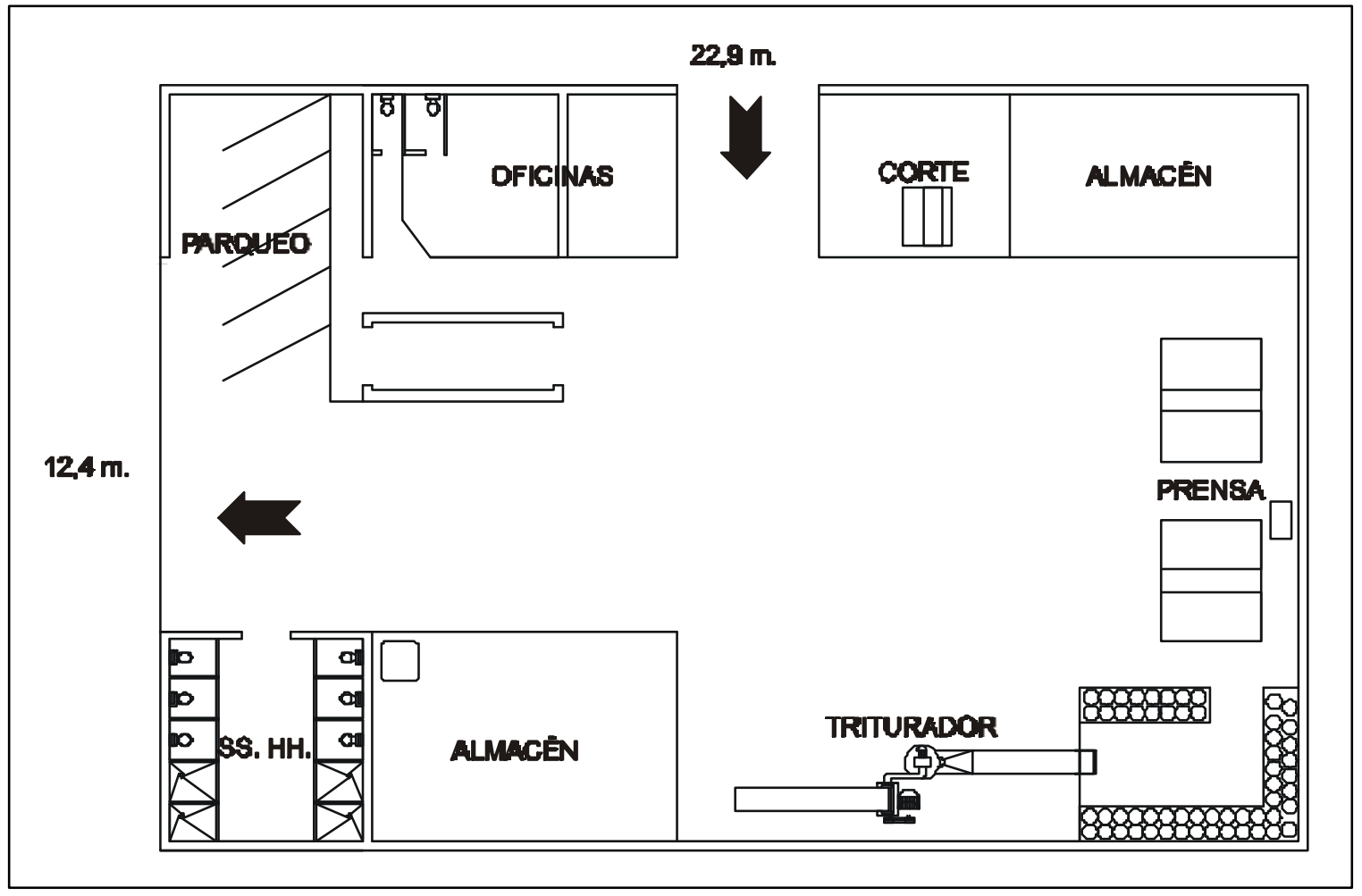

Figura 8. Distribución de la planta 
Cuadro 6. Inversión inicial (en dólares americanos)

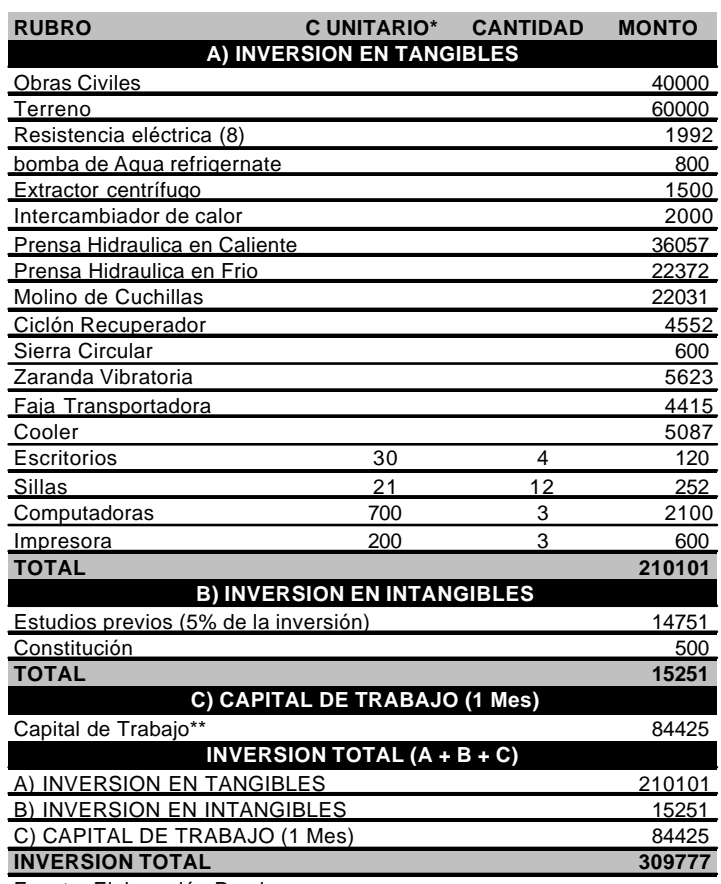

FUERSIONTOTAL

(*) Incluye IGV

$\left.{ }^{* \star}\right)$ Se ha determinado un mes de costos totales sin incluir la depreciación, ya que sólo es necesario financiar los costos del primer mes debido a que con sus utilidades se financia el mes siguiente
Cuadro 7. Consumo de energía

\begin{tabular}{|c|c|c|c|c|}
\hline EQUIPOS & $\begin{array}{c}\text { Potencia } \\
\text { (KW) }\end{array}$ & \begin{tabular}{|c|} 
Tiempo de \\
Operación \\
(h)
\end{tabular} & $\begin{array}{c}\begin{array}{c}\text { Consumo } \\
\text { de energía } \\
\text { (KWh) }\end{array} \\
\end{array}$ & $\begin{array}{l}\text { Corriente } \\
\text { eléctrica } \\
\text { (A) }\end{array}$ \\
\hline $\begin{array}{l}\text { Molino de } \\
\text { cuchillas }\end{array}$ & 22,5 & 2 & 45 & 100 \\
\hline $\begin{array}{l}\text { Prensa } \\
\text { hidráulica (2) }\end{array}$ & 11,25 & 9 & 202,5 & 100 \\
\hline $\begin{array}{l}\text { Resistencia } \\
\text { eléctrica (8) }\end{array}$ & 20 & 8 & 160 & 90 \\
\hline $\begin{array}{l}\text { Bomba de } \\
\text { Aqua-Refriger. }\end{array}$ & 0,75 & 10 & 15 & 7,0 \\
\hline $\begin{array}{l}\text { Zaranda } \\
\text { vibratoria }\end{array}$ & 0,75 & 2 & 1,5 & 3,5 \\
\hline $\begin{array}{l}\text { Extractor } \\
\text { centrífugo } \\
\end{array}$ & 1,5 & 2 & 3 & 7 \\
\hline $\begin{array}{l}\text { Intercambiador } \\
\text { de calor }\end{array}$ & 0,375 & 4 & 3 & 3,5 \\
\hline \begin{tabular}{l|} 
Faja \\
transportadora
\end{tabular} & 0,75 & 10 & 15 & 7 \\
\hline Sierra circular & 1.5 & 5 & 15 & 14 \\
\hline Iluminación & 2.3 & 10 & 23 & 11 \\
\hline \multicolumn{3}{|c|}{ CONSUMO TOTAL } & 483 & 343 \\
\hline
\end{tabular}

Cuadro 8. Costos indirectos (en dólares americanos)

\begin{tabular}{|l|r|r|}
\hline RUBRO & MENSUAL & \multicolumn{1}{c|}{ ANUAL } \\
\hline Jefe de Planta & 424 & 5088 \\
\hline Depreciación & 966 & 11592 \\
\hline Energía eléctrica & 1167 & 14004 \\
\hline Aqua & 38 & 456 \\
\hline Teléfono & 60 & 720 \\
\hline Imprevistos 5\% & 133 & 1596 \\
\hline TOTAL & $\mathbf{2 7 8 8}$ & $\mathbf{3 3 4 5 6}$ \\
\hline
\end{tabular}

Fuente : Elaboración propia

Cuadro 9. Costo de mano de obra de producción (en dólares americanos).

\begin{tabular}{|c|c|c|c|c|c|c|c|}
\hline RUBRO & BASICO (S/.) & BASICO (\$) & BBSS & APORTACIONES & C. UNIT.(\$) & CANTIDAD & MONTO (\$) \\
\hline Jefe de Planta & \begin{tabular}{r|}
1000 \\
\end{tabular} & 303 & \begin{tabular}{|r|}
76 \\
\end{tabular} & $\begin{array}{rr}45 \\
\end{array}$ & 424 & 1 & 424 \\
\hline Obrero & 700 & 212 & 53 & 32 & 297 & 10 & 2970 \\
\hline TOTAL & & & & & & & 3394 \\
\hline
\end{tabular}

Fuente : Elaboración propia

$\left({ }^{*}\right)$ BBSS corresponde $25 \%$ y Aportaciones $15 \%$, ambos del básico

Cuadro 10. Depreciación del costo indirecto de producción (en dólares americanos).

\begin{tabular}{|l|r|r|r|r|}
\hline \multicolumn{1}{|c|}{ RUBROS } & $\begin{array}{r}\text { MONTO } \\
\text { INICIAL }\end{array}$ & MESES & RESCATE & MENSUAL \\
\hline Obras Civiles & 40000 & 360 & 0 & 111 \\
\hline Computadora & 700 & 60 & 0 & 12 \\
\hline Impresora & 200 & 60 & 0 & 3 \\
\hline Prensa Hidraulica en & 36057 & 120 & 0 & 300 \\
\hline Prensa Hidraulica en & 22372 & 120 & 0 & 186 \\
\hline Molino de Cuchillas & 22031 & 120 & 0 & 184 \\
\hline Ciclón Recuperador & 4552 & 120 & 0 & 38 \\
\hline Sierra Circular & 600 & 120 & 0 & 5 \\
\hline Zaranda Vibratoria & 5623 & 120 & 0 & 48 \\
\hline Faia Transportadora & 4415 & 120 & 0 & 37 \\
\hline Cooler & 5087 & 120 & 0 & 42 \\
\hline TOTAL & & & 966 \\
\hline
\end{tabular}

Fuente : Elaboración propia 
Cuadro 11. Costo de producción (en dólares americanos)

\begin{tabular}{|l|r|r|}
\hline RUBRO & MENSUAL & \multicolumn{1}{c|}{ ANUAL } \\
\hline Materia Prima & 758 & 9096 \\
\hline Mano de Obra Obreros & 2970 & 35640 \\
\hline Costos indirectos & 2788 & 33456 \\
\hline TOTAL & 6516 & 78192 \\
\hline
\end{tabular}

$(*) 1$ TM de Tetra Pak= US $\$ 15.00$
Cuadro 12. Costo de personal administrativo (en dólares americanos)

\begin{tabular}{|l|r|r|r|r|r}
\hline RUBRO & $\begin{array}{c}\text { BASICO } \\
\text { (S/.) }\end{array}$ & $\begin{array}{c}\text { BASICO } \\
\text { (\$) }\end{array}$ & BBSS* $^{\star}$ & $\begin{array}{c}\text { APORTACI } \\
\text { ONES* }\end{array}$ & $\begin{array}{c}\text { TOTAL } \\
\text { (\$) }\end{array}$ \\
\hline Gerente Genera & 1500 & 455 & 114 & 68 & 637 \\
\hline Secretaria & 750 & 242 & 61 & 36 & 339 \\
\hline TOTAL & \multicolumn{3}{|r}{} & & 976 \\
\hline
\end{tabular}

(*) BBSS corresponde $25 \%$ y Aportaciones $15 \%$, ambos del Básico

Cuadro 13. Depreciación de los costos administrativos (en dólares americanos)

\begin{tabular}{|c|c|c|c|c|c|c|c|}
\hline \multirow{2}{*}{ RUBROS } & \multirow{2}{*}{$\begin{array}{l}\text { MONTO } \\
\text { INICIAL }\end{array}$} & \multirow{2}{*}{ CANTIDAD } & \multirow{2}{*}{$\begin{array}{l}\text { MONTO } \\
\text { TOTAL }\end{array}$} & \multirow{2}{*}{ AÑOS } & \multirow{2}{*}{ RESCATE } & \multicolumn{2}{|c|}{ DEPRECIACIÓN } \\
\hline & & & & & & ANUAL & MENSUAL \\
\hline Escritorios & 30 & 4 & 120 & 10 & 0 & 3 & 0 \\
\hline Sillas & 21 & 12 & 252 & 10 & 0 & 2 & 0 \\
\hline Computadoras & 700 & 2 & 1400 & 5 & 0 & 140 & 12 \\
\hline Impresora & 200 & 3 & 600 & 5 & 0 & 40 & 3 \\
\hline TOTAL & & & & & & 185 & 15 \\
\hline
\end{tabular}

Fuente: Elaboración propia

Cuadro 14. Costos administrativos (en dólares americanos)

\begin{tabular}{|l|r|r|}
\hline RUBROS & MENSUAL & ANUAL \\
\hline Gerente General & 637 & 7644 \\
\hline Secretaria & 339 & 4068 \\
\hline Contador (Tiempo Parcial) & 242 & 2904 \\
\hline Depreciación Utiles de Oficina & 15 & 185 \\
\hline Consumo de energia & 20 & 240 \\
\hline Consumo de Agua & 10 & 120 \\
\hline Teléfono & 90 & 1080 \\
\hline Transporte & 76 & 912 \\
\hline Imprevistos 5\% & 71 & 852 \\
\hline TOTAL & $\mathbf{1 5 0 0}$ & $\mathbf{1 8 0 0 5}$ \\
\hline
\end{tabular}

Cuadro 15. Costo total anual (en dólares americanos)

\begin{tabular}{|l|r|}
\hline RUBRO & MONTO (en US \$) \\
\hline Costos de Producción & 78192 \\
\hline Costos de Administración & 18005 \\
\hline TOTAL & $\mathbf{9 6 1 9 7}$ \\
\hline
\end{tabular}

Fuente : Elaboración propia

Cuadro 16. Depreciación total anual (en dólares americanos)

\begin{tabular}{|l|r|}
\hline DEPRECIACIÓN & \multicolumn{1}{|c|}{ MONTO } \\
\hline Costo indirecto de producción & 11587 \\
\hline Costos administrativos & 185 \\
\hline TOTAL & $\mathbf{1 1 7 7 2}$ \\
\hline
\end{tabular}

Fuente : Elaboración propia 
Cuadro 17. Ingresos (en dólares americanos)

\begin{tabular}{|l|r|c|}
\hline RUBRO & MONTO & UNIDAD \\
\hline Precio por plancha & 6 & $\$$ /plancha \\
\hline Producción Diaria & 126 & plancha/dia \\
\hline Ingreso Diario & 756 & $\$ /$ dia \\
\hline 1 mes & 25 & dias/mes \\
\hline Ingreso Mensual & 18900 & $\$ /$ mes \\
\hline 1 año & 12 & meses/año \\
\hline Ingreso Anual & 226800 & $\$ /$ año \\
\hline
\end{tabular}

Fuente : Elaboración propia

Cuadro 18. Flujo de caja (en dólares americanos)

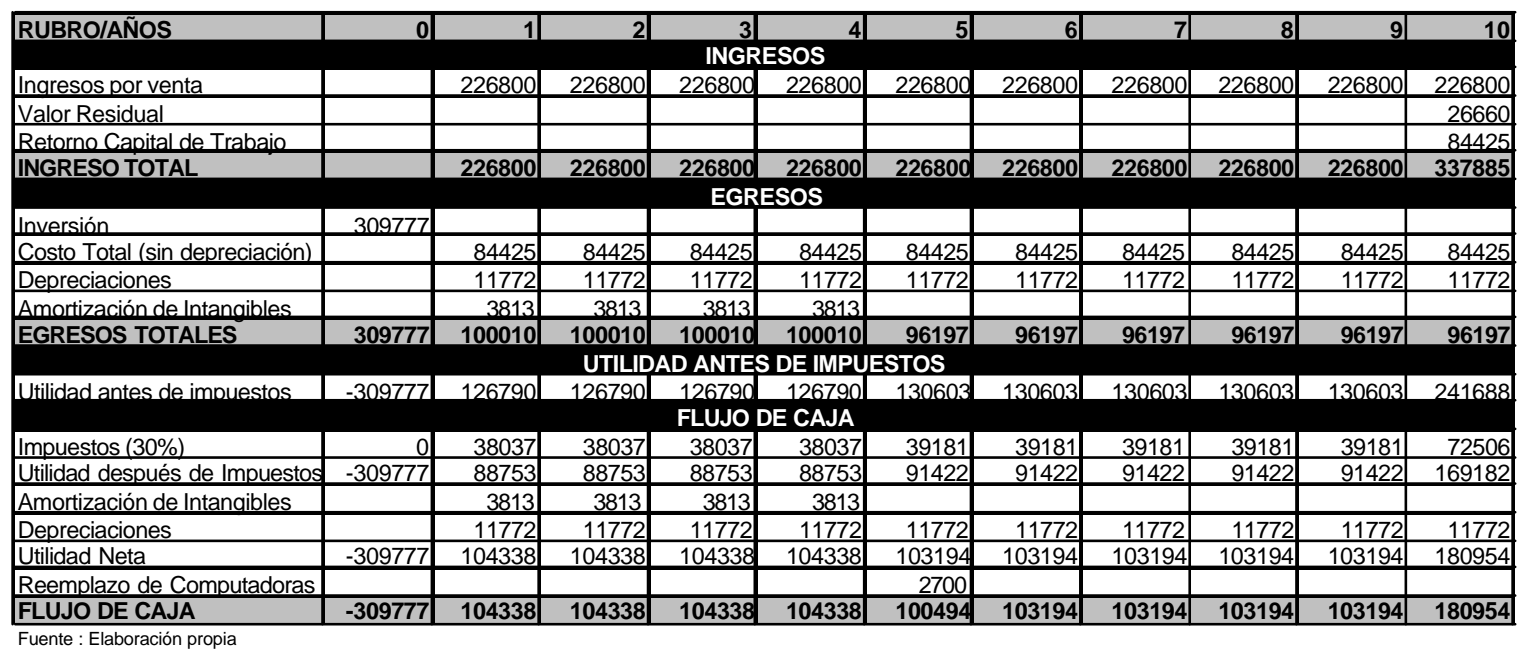

Cuadro 19. Evaluación económica

\begin{tabular}{|l|r|}
\hline INDICADOR & RESULTADO \\
\hline VAN (en Dólares) & 300271 \\
\hline TIR & $32 \%$ \\
\hline B/C & 2.37 \\
\hline Periodo de Recupero & 3 años \\
\hline TASA DE CORTE (\%) & 12 \\
\hline \multicolumn{2}{|l}{ Fuente: Elaboración propia }
\end{tabular}

(VAN), la Tasa de Interna de Retorno (TIR) y, el valor Beneficio/Costo (B/C) mostrados en el Cuadro 19.

\section{ANÁLISIS Y DISCUSIÓN}

En el estudio se parte de una disposición en "U" de las etapas del proceso. En realidad, no existe ninguna disposición perfecta, se habrá tenido que sacrificar algu- nas características para favorecer otras. En ese sentido, es necesario enfocar los beneficios que se recibirán en la disposición. Para ello es conveniente eliminar obstáculos en la trayectoria de los materiales.

La capacidad de planta se determina según la demanda del mercado, en el estudio se considera una producción diaria de 126 planchas. Si esta producción se duplica se emplearía un turno adicional y el costo de producción disminuiría en un $10 \%$. 
El cuello de botella en la cadena productiva se localiza en la prensa hidráulica; ya que cada plancha implica 4 a 4,5 minutos de duración y en las 8 horas de trabajo se producen 126 planchas, el cual es la capacidad máxima tomada en el estudio.

Al respecto, es conveniente realizar modificaciones en la prensa hidráulica de manera que simultáneamente se pueda comprimir al menos 3 planchas, a fin de garantizar el incremento de la producción.

\section{CONCLUSIONES}

El sector industrial debe impulsar el reciclaje de envases tetra pak para la creación de pequeñas empresas, considerando la disponibilidad de residuos.

Con el análisis económico se demuestra la viabilidad de la implementación de la planta de reciclaje de envases tetra pak.

La fabricación de planchas de aglomerados tiene múltiples aplicaciones en la mueblería y en la construcción, por la diferencia de costos con la madera.

Es posible lograr en la comunidad de segregadores informales el comercio de envases usados de tetra pak, al inicio con un precio de US \$15/TM, base para determinar el costo de materia prima.
Es necesaria la recuperación de los envases usados de tetra pak por dos razones principales: la contaminación que provocan y el valor económico que representan para el reciclado.

\section{BIBLIOGRAFÍA}

1. CONAMA (2002). Reciclaje Masivo - Acción Comunitaria. En: www.conama.cl/rm/568/article2272.html

2. CONAMA (2002). Reciclaje Masivo - Recolección. En:www.conama.cl/rm/568/article-1342.html

3. Ciudad Viva (2002). Campaña. En:www.ciudadviva.cl/ basura/tpak.html

4. Jaan Koel (2002). Foundations for Sustainability. En: www.rco.on.ca/intro/upcoming/23_2002/ J_Koel.doc

5. Tetra Pak (2003). Información de la Empresa Tetra Pak. En: www.tetrapak.com.pe

6. Umweltbundesamt (2003). Facts and Figures. En: www.umweltbundesamt.de/uba-info-daten-e/ index.htm

7. Ulrich, Gael D. (1998). Diseño y Economía de los Proceso de Ingeniería. Editorial Interamericana. México. 